\title{
Bistatic configurational analysis of ultra-wideband antenna for detection applications
}

\author{
Jawad Ali ${ }^{1}$, Noorsaliza Abdullah ${ }^{2}$, Roshayati Yahya ${ }^{3}$, Ezri Mohd ${ }^{4}$, Ariffuddin Joret ${ }^{5}$, \\ Norshidah Katiran ${ }^{6}$ \\ 1,2,3,4,5, Department of Communication Engineering, Faculty of Electrical and Electronic Engineering, Universiti Tun \\ Hussein Onn Malaysia (UTHM), Johor, Malaysia \\ ${ }^{1}$ Electrical Engineering Department, COMSATS University Islamabad (CUI), Lahore Campus, Pakistan
}

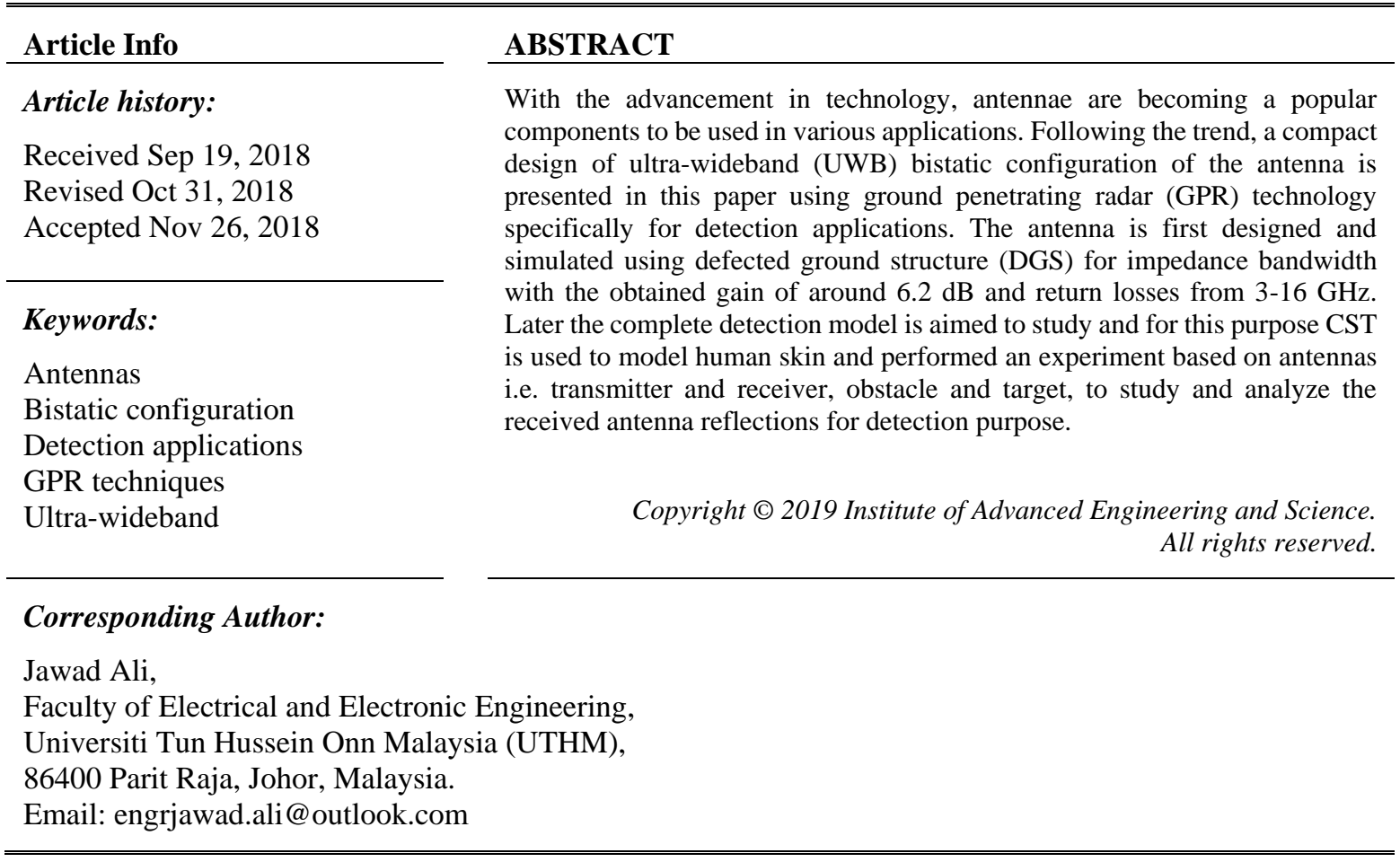

\section{INTRODUCTION}

Ultra-wideband (UWB) systems are gaining popularity in a recent couple of years. This radio technology mainly refers to a system, which has very large operating bandwidth. In 1893 the first experiment on UWB was conducted for the production of electromagnetic waves [1] since it was not available commercially at that time so few experiments were reported by that time. Later the state department of the United State formally unlicensed the $7.5 \mathrm{GHz}$ bandwidth to commercialize the researcher's contributions for industrial and scientific experimentation. The frequency band allocated for UWB ranges from 3.1-10.6 GHz with a number of significant advantages such as the consumption of power is less compared to other microwave frequency bands, the cost of implementation is minimum, and as it also has a lower frequency within the range so UWB also has the capability of obstacle penetration [2]. Due to these achievable advantages of UWB, it has a number of applications in the field of communications, and radars such as ground penetrating radar (GPR), imaging and positioning [3].

Moreover, a number of GPR applications uses UWB technology, mainly in military and civilian applications such as for the detection of landmines and the localization of buried victims under the rubble or in an opaque environment [4]. The main components of GPR are receiver and transmitter antennas that must be involved in the process for any application, so for the implementation of UWB antenna technology in mentioned GPR applications many antennas such as vivaldi, planar, horn, and bow-tie antennas have been reported in the previous study. Since the reported designs are fulfilling the requirements such as gain, directivity, frequency bandwidth, and the most important design complexity for GPR applications [5-6]. In this 
paper, an arc shaped UWB planar antenna using FR-4 as a dielectric substrate is reported. The gain and bandwidth of the designed antenna are obtained around $6 \mathrm{~dB}$ and $13 \mathrm{GHz}$ respectively. The design is achieved using a modified defected ground structure (DGS) method [7]. To fulfill the purpose of detection through wall an experiment is modeled and simulated using CST Microwave Studio. In this experiment, a bistatic antenna is introduced to study the reflections obtained from the target and the subsequent results are then presented for further analysis.

The remainder of the paper is organized as follows; a complete antenna design with a simulation of the bistatic model including wall as an obstacle and human skin as a target using GPR technology is discussed in Section 2. While the analysis based on obtained results is presented in Section 3. Final section summarized the discussion in the form of a conclusion.

\section{SIMULATION MODEL AND DESIGN}

The proposed antenna and the bistatic configuration for ground penetrating radar (GPR) model are designed and simulated using CST Microwave Studio transient solver technique with the fixed impedance of $50 \mathrm{ohms}$. This section carried out the discussion about a design of ultra-wideband (UWB) antenna and its simulation based on an experiment of bistatic antenna model for detection applications.

To implement the design of UWB antenna ranges from 3-10 GHz for detection application using GPR technology, an arc-shaped planar antenna using FR-4 as a dielectric substrate with $\mathrm{Er}=4.2$ is designed in this paper. To achieve the design a partially circular slot is introduced towards the minor axis of the elliptical patch and optimize the position of the circle for the tuning of scattering parameter. The design of UWB arc antenna is demonstrated in Figure 1, which shows the compactness in antenna size. As there is a concept that antenna size is greater at a lower frequency, so the antenna size is matched at the lower band of UWB frequency components using half ground method [8]. To achieve the gain of $6 \mathrm{~dB}$, a DGS method is introduced with the slots at the different areas on the ground as shown in Figure 1. In this case, a DGS method is used to make the ground plane half, in order to match the bandwidth throughout the UWB frequency band and for directional radiation. Table 1 shows the material and dimension of the substrate, ground, the minor and major axis of the arc along with excitation port properties.

After the successful simulation of the proposed antenna. The simulation-based experiment for bistatic antenna model using GPR concept is carried out to observe the detection capability of the proposed antenna. Basically, bistatic antennas are those in which the position of transmitting antenna is fixed while varying the position of receiver antenna [3] in order to receive the proper reflections.

Table 1. Arc Antenna Design Parameters

\begin{tabular}{cc}
\hline Parameter & Dimensions $(\mathrm{mm})$ \\
\hline Copper Patch Major arc axis & 27 \\
Copper Patch Minor arc axis & 17.28 \\
FR-4 Substrate (LxW) & $40 \times 35$ \\
Copper Ground (L1xW) & $20 \times 35$ \\
Waveguide Port (HxW1) & $3.95 \times 7.14$ \\
\hline
\end{tabular}

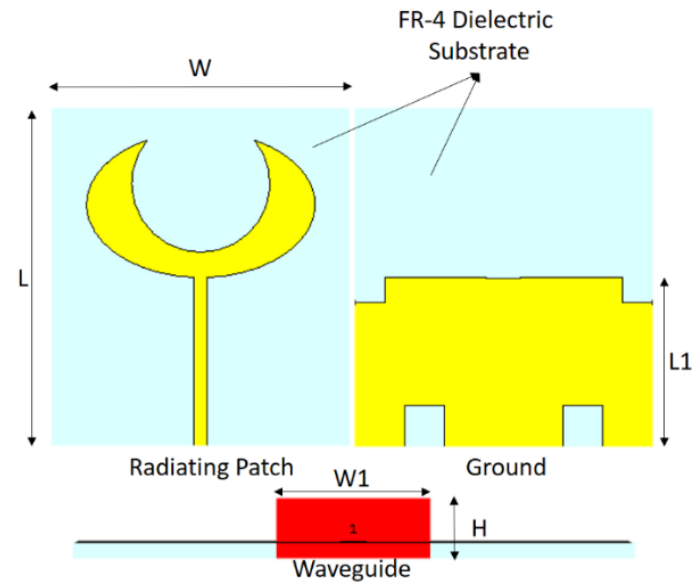

Figure 1. Proposed planar antenna design 
The complete simulation scenario of bistatic configuration is illustrated in Figure 2. In the figure two antennas are placed at a certain distance from each other, the transmitter position is fixed to transmit the electromagnetic wave and the position of the receiver is varied to receive reflections.

These antennas are placed at a distance from an obstacle, which is chosen to be as a concrete slab of thickness $9 \mathrm{~cm}$ to test the penetration efficiency of antennas. On the other hand, a targeted human skin structure is placed behind the concrete obstacle. The distance between the antennas and obstacle, also between the obstacle and target can be determined as 'Antenna to Wall' and 'Wall to Target' respectively. The dielectric constant of concrete available in the material library of CST Microwave studio is $\varepsilon_{\mathrm{r}}=5.46$ for the complete UWB band, whereas the dielectric of skin varies from $\mathrm{Er}=36$ to $\mathrm{Er}=26$ from lower to higher frequency. The analysis of the obtained results is the part of the study in the result and discussion section.

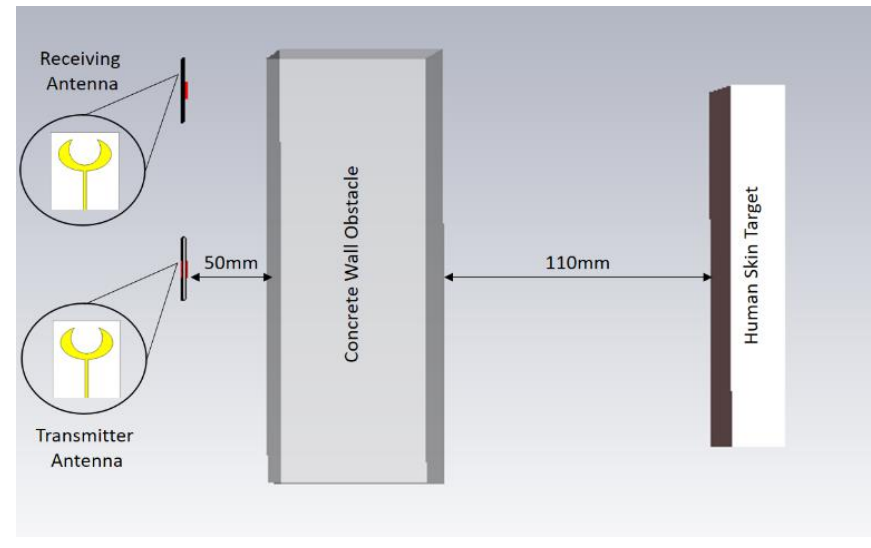

Figure 2. Simulation-based experimental model

\section{RESULTS AND ANALYSIS}

The obtained results of the proposed antenna and bistatic GPR model is analyzed in this section. The scattering parameter $\left(S_{11}\right)$ and mutual coupling $\left(S_{21}\right)$ of the design for bandwidth coverage of UWB bistatic antenna which is greater than the allocated band are shown in Figure 3(a). The obtained $S_{11}$ ranges from 3 to $16 \mathrm{GHz}$ with a bandwidth of $13 \mathrm{GHz}$ which provides $5.5 \mathrm{GHz}$ more band. Another factor of UWB antenna design for GPR applications is the gain, which evaluates the penetration efficiency. Figure 3(b) presents the major lobe gain of the proposed antenna to be $6.21 \mathrm{~dB}$ which is observed at $6.5 \mathrm{GHz}$. The radiation pattern at the same frequency is also focusing in a directional manner. Thus, these obtained results can be helpful for detecting target behind the obstacle.

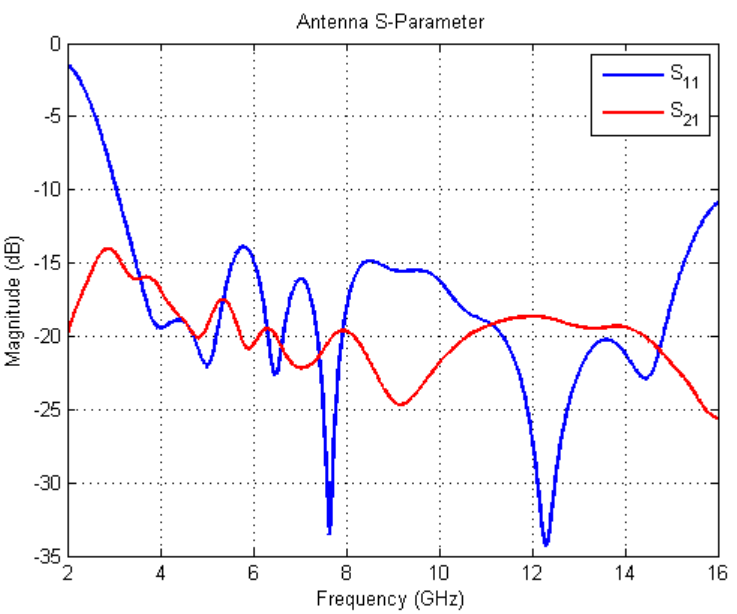

(a)

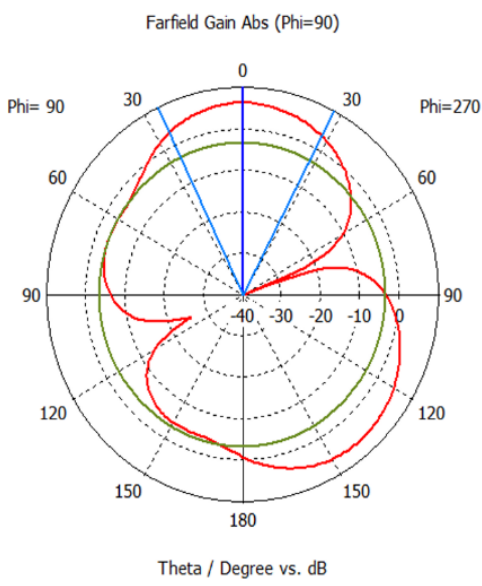

(b)

Figure 3. Arc antenna (a) Return loss from 3-16 GHz (b) Radiation with main lobe gain $6.21 \mathrm{~dB}$ 
To further the analysis, an experiment for bistatic antenna model using GPR technique is conducted for detection applications as discussed earlier. The results of this simulation experiment are analyzed in the form of scattering parameters, and time reflections obtained from transmitting antenna, obstacle and skin target towards receiving antenna. Figure 4 shows the obtained results when electromagnetic wave passed through the obstacle and reflected energy is being received by the receiving antenna place at a certain distance from the transmitter. The $S_{11}$ of the antenna when there is no obstacle in front of the receiver antenna is compared with the results when the complete detection model is simulated as shown in Figure 4(a). It can be seen from the figure that obtained results in the presence of obstacle and target caused disturbance in $\mathrm{S}_{11}$ but around $4 \mathrm{GHz}$, a significant resonance is noted which is mainly due to backscattering of the wall. Whereas the same results can be observed in Figure 4(b) which is basically the mutual coupling to transmitting and receiving antenna in the form of $S_{21}$ again the same resonance is observed, it also demonstrates the presence of an object which causes disruption in antenna reflections.

The time reflections of the transmitted and received signal can also be observed in Figure 5(a), from which it can be seen clearly that the maximum signal shows the presence of antenna but the small reflections also indicate the obstacle and targeted object.

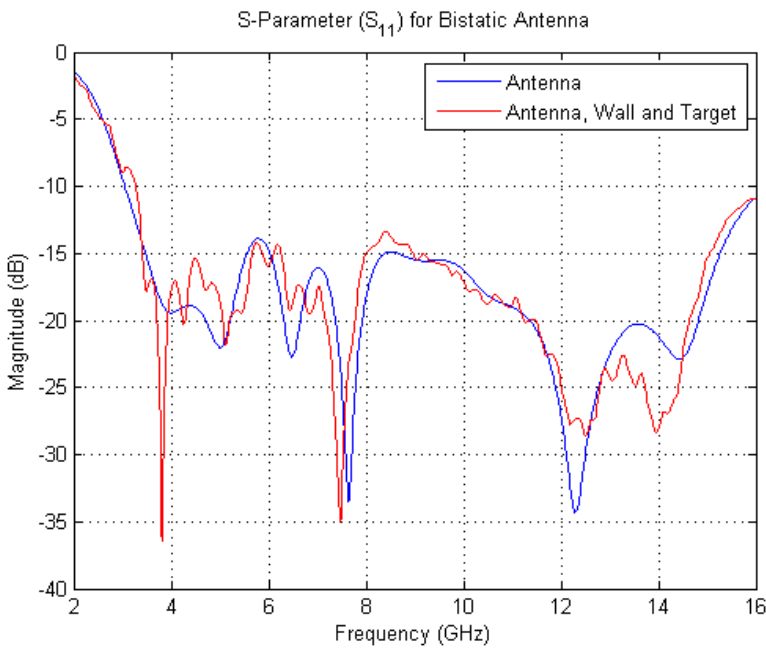

(a)

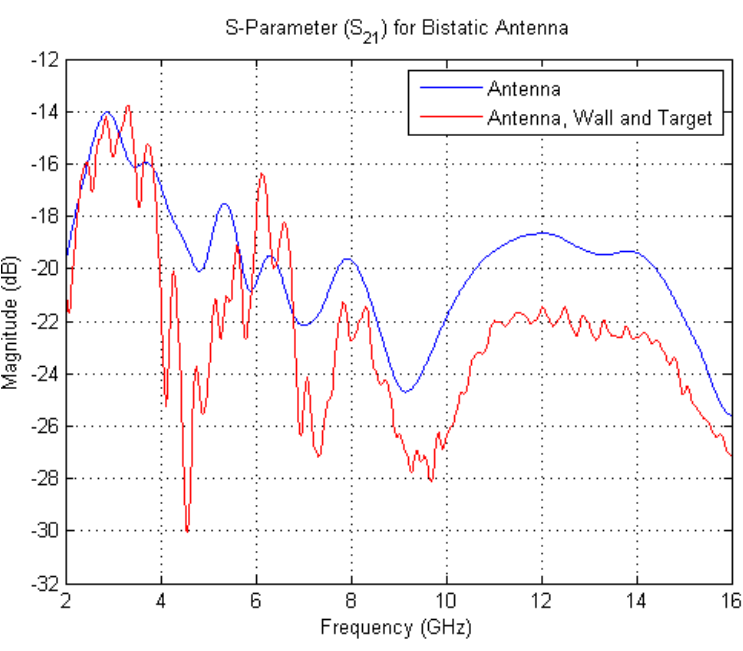

(b)

Figure 4. Scattering parameter of antennas (a) $S_{11}$ reflections (b) $S_{21}$ reflections

Based on the results in the form of time reflections, it is obvious that antenna is receiving scattering reflections from the object behind the obstacle. Thereafter, the obtained scattering parameters are also analyzed in MATLAB for the computation of radar cross section (RCS) based 2D imaging, as shown in Figure 5(b) where the disrupting wave signals representing the presence of target and wall. Based on the time $t$ of the received reflections, one can easily calculate the distance of the object using Equation (1) and (2) [9]:

$$
\begin{aligned}
& d=v \frac{t}{2} \\
& v=\frac{c}{\sqrt{\varepsilon_{r}}}
\end{aligned}
$$

In these equations, $v$ is the propagation velocity which is the ratio of the speed of light $c$ to dielectric value $\varepsilon_{r}$ of the medium where the wave is traveling at the defined time.

The obtained results are demonstrating the presence of an object to be detected but it is difficult to differentiate between them. The main reason for this noisy image is basically a data, as when exported from CST it is in RAW format. In order to make the image more clear different digital signal processing techniques such as interpolation, filtering needs to be implemented [11] which will be the future part. 


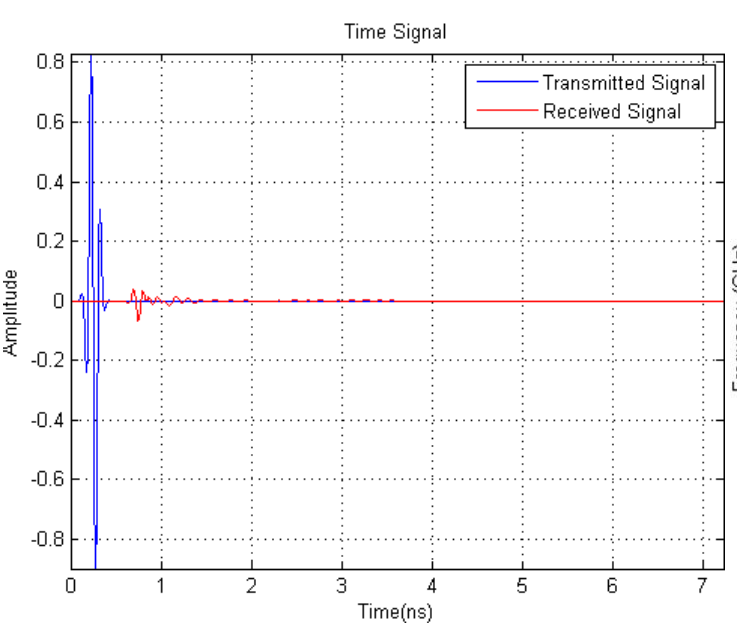

(a)

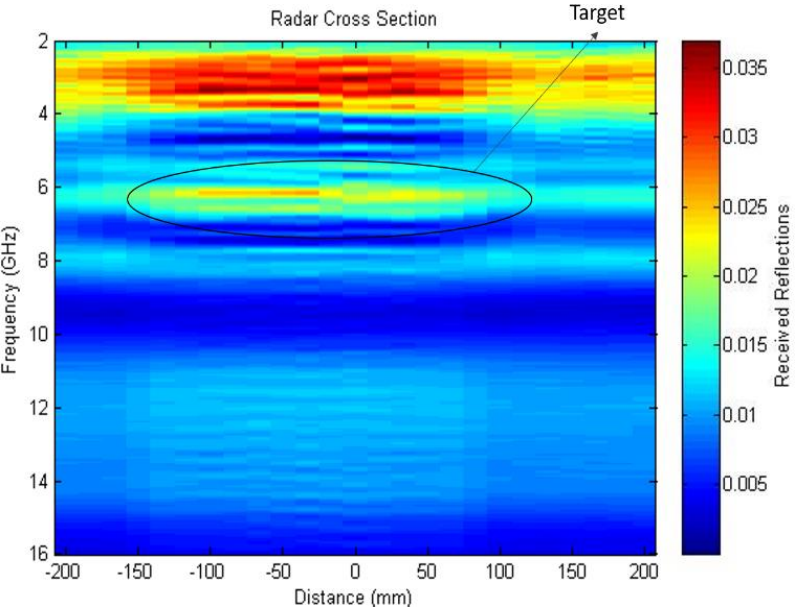

(b)

Figure 5. Bistatic GPR (a) Time signal (b) Radar cross section based 2D image of experimentation

\section{CONCLUSION}

An ultra-wideband (UWB) planar arc shaped antenna design has been proposed and experimented for detection application. The compactness of the design is carried out using FR-4 dielectric substrate. The obtained gain as a result of directional radiation is $6.21 \mathrm{~dB}$ with the bandwidth of $13 \mathrm{GHz}$. Thereafter, an experimental model is then designed and simulated, which consisted of two antennae one as a transmitter and another as a receiver with an obstacle between antennae and the targeted human skin. The results of this experiment are then analyzed and presented in the form of RCS based 2D imaging to study the received reflections from antenna, obstacle, and target. Thus this demonstration provided with an information that UWB antenna with bistatic configuration can be a useful system for the detection operation in ground penetrating radar (GPR) applications.

\section{ACKNOWLEDGMENTS}

The authors would like to acknowledge ORICC Universiti Tun Hussein Onn Malaysia (UTHM) for supporting this work under TEIR 1 research grant with VOT U860.

\section{REFERENCES}

[1] M. Ghavami, L. B. Michael, and R. Kohno, Ultra-Wideband Signals and Systems in Communication Engineering, New York: Wiley, 2007, pp. 4-17.

[2] D. Valderas, X. Chen, C. Ling, J. I. Sancho, and D. Puente, Ultrawideband antennas: Design and applications, London: Imperial College Press, 2010, pp. 1-13.

[3] W. Kang et al., "A study of antenna configuration for bistatic ground-penetrating radar," in 2016 16th International Conference on Ground Penetrating Radar (GPR), pp. 1-4.

[4] C. Warren and A. Giannopoulos, "Experimental and Modeled Performance of a Ground Penetrating Radar Antenna in Lossy Dielectrics," IEEE J. of Selected Topics in Applied Earth Observations and Remote Sensing, vol. 9, pp. 2936, Jan. 2016.

[5] E. A. Etellisi, M. A. Elmansouri and D. S. Filipovic, "Wideband Monostatic Simultaneous Transmit and Receive (STAR) Antenna," IEEE Trans. on Antennas and Propagation, vol. 64, pp. 6-15, Jan. 2016.

[6] G. Pochanin, S. Masalov, I. Pochanina, L. Capineri, P. Falorni and T. Bechtel, "Modern trends in development and application of the UWB radar systems," in 2016 8th International Conference on Ultrawideband and Ultrashort Impulse Signals (UWBUSIS), pp. 7-11.

[7] R.A. Pandhare, P.L. Zade, and M.P. Abegaonkar, "Miniaturized microstrip antenna array using defected ground structure with enhanced performance," Engineering Science and Technology, an International Journal, vol. 19, pp. 1360-1367, Apr. 2016.

[8] S. Koziel and A. Bekasiewicz, "A Structure and Simulation-Driven Design of Compact CPW-Fed UWB Antenna," IEEE Antennas and Wireless Propagation Lett, vol. 15, pp. 750-753, 2016.

[9] D. J. Daniels, Ground Penetrating Radar, London: The Institution of Engineering and Technology, 2004, ch. 4.

[10] X. Zeng, A. Fhager, M. Persson, P. Linner and H. Zirath, "Accuracy Evaluation of Ultrawideband Time Domain Systems for Microwave Imaging," IEEE Trans. on Antennas and Propagation, vol. 59, pp. 4279-4285, Nov. 2011. 
[11] ZYCH, Mariusz, "Ground penetrating radar simulations of non-homogeneous soil with CST Studio Suite," Przegląd Elektrotechniczny, vol. 89, pp. 182-185, 2013.

\section{BIOGRAPHIES OF AUTHORS}

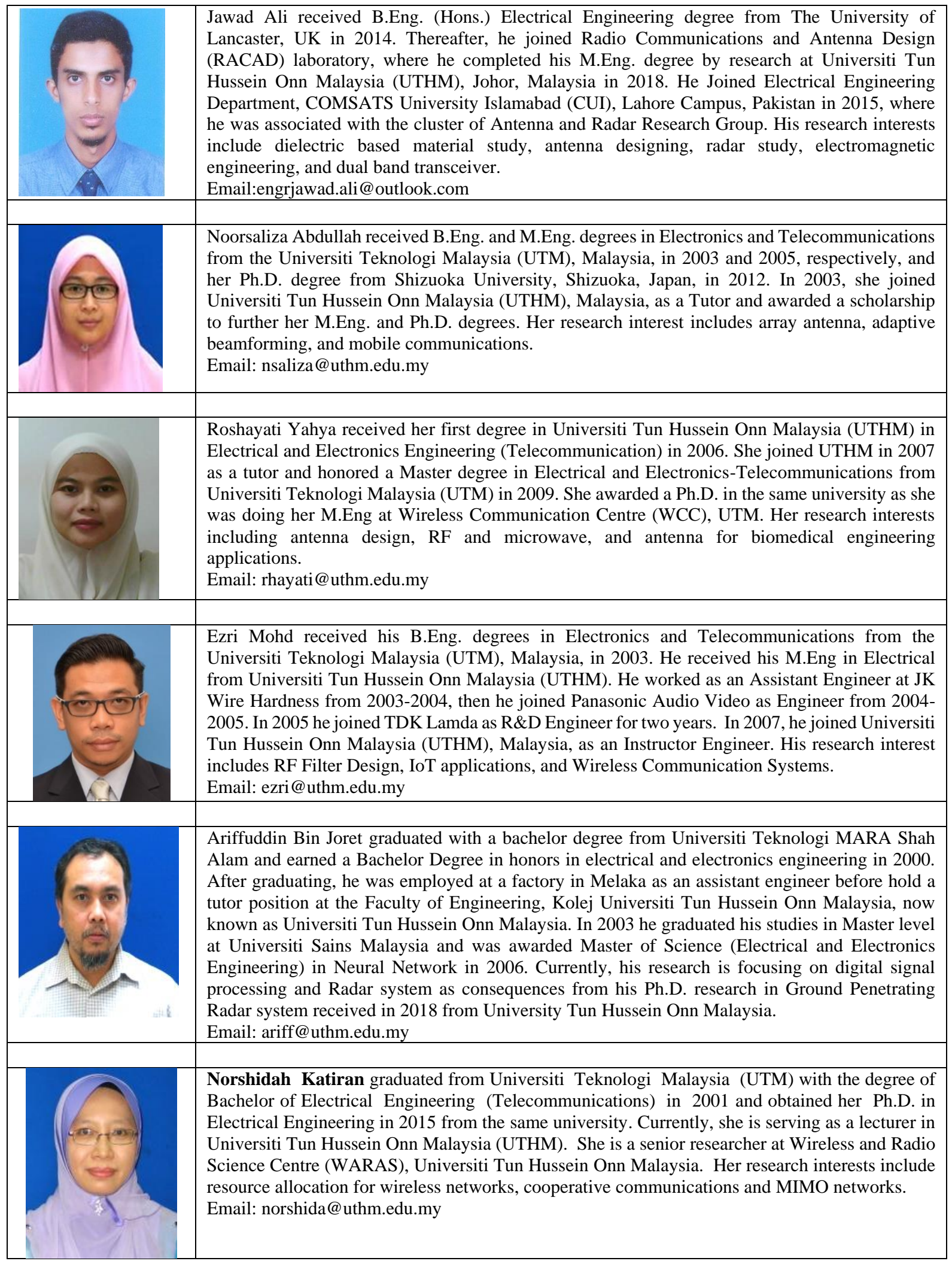

